Relations industrielles

Industrial Relations

Gouvernance des régimes complémentaires de retraite, relations du travail et conflits de rôle : une enquête québécoise Governance of Supplementary Pension Plans, Labour Relations and Role Conflicts: A Quebec Study Gobernanza de regímenes complementarios de jubilación, relaciones laborales y conflictos de rol: un estudio quebequense

\author{
Daniel Coulombe, Esther Déom, Frédéric Hanin et Annette Hayden
}

Volume 72, numéro 2, printemps 2017

URI : https://id.erudit.org/iderudit/1040400ar

DOI : https://doi.org/10.7202/1040400ar

Aller au sommaire du numéro

\section{Éditeur(s)}

Département des relations industrielles de l’Université Laval

\section{ISSN}

0034-379X (imprimé)

1703-8138 (numérique)

Découvrir la revue

Citer cet article

Coulombe, D., Déom, E., Hanin, F. \& Hayden, A. (2017). Gouvernance des régimes complémentaires de retraite, relations du travail et conflits de rôle : une enquête québécoise. Relations industrielles / Industrial Relations, 72(2), 246-269. https://doi.org/10.7202/1040400ar
Résumé de l'article

Cette recherche vise à étudier la présence de conflits de rôle au sein des comités de retraite au Québec. Elle met en relation la gouvernance des régimes de retraite et la présence de conflits de rôle entre la représentation collective et la responsabilité fiduciaire du point de vue des membres de comités de retraite. Nous exploitons une particularité de la gestion québécoise, soit l'obligation de créer un comité de retraite sur lequel siègent des membres représentant l'employeur, les syndicats, les travailleurs, les retraités, ainsi que des membres indépendants. À l'aide d'une méthodologie mixte par questionnaire et par entrevue, il a été possible d'analyser les sources et la nature des conflits.

Contrairement à nos anticipations, notre étude ne démontre pas de conflits systématiques causés par la coexistence du système de relations du travail et de la responsabilité fiduciaire des régimes complémentaires de retraite. Les tensions reliées aux relations du travail sont minimes. Nous avons bien identifié quelques enjeux, notamment au niveau du financement des régimes, de la viabilité des régimes, qui relèvent de la confusion des rôles.

Toutefois ces éléments ne sont pas perçus comme des sources de tensions, mais plutôt comme des enjeux à discuter. La qualité de la gouvernance des régimes de retraite dépend des ressources investies par les acteurs des relations du travail dans l'accompagnement des membres des comités de retraite. La recherche sur les conflits de rôle dans la gouvernance des régimes de retraite au Québec montre que la gestion de ces conflits dépend, dans une large mesure, de la qualité de la législation sur l'administration des régimes complémentaires de retraite au Québec et, également, des ressources consacrées à la formation des membres des comités de retraite et des participants aux relations du travail.
Tous droits réservés @ Département des relations industrielles de l’Université Laval, 2017
Ce document est protégé par la loi sur le droit d'auteur. L’utilisation des services d’Érudit (y compris la reproduction) est assujettie à sa politique d'utilisation que vous pouvez consulter en ligne.

https://apropos.erudit.org/fr/usagers/politique-dutilisation/ 


\title{
Gouvernance des régimes complémentaires de retraite, relations du travail et conflits de rôle: une enquête québécoise
}

\author{
Daniel Coulombe, Esther Déom, Frédéric Hanin \\ et Annette Hayden
}

\begin{abstract}
Cette recherche étudie la présence de conflits de rôle au sein des comités de retraite au Québec. Elle met en relation la gouvernance des régimes de retraite et la présence de conflits de rôle entre la représentation collective et la responsabilité fiduciaire du point de vue des membres de comités de retraite. À l'aide d'une méthodologie mixte par questionnaire et par entrevue, il a été possible d'analyser les sources et la nature des conflits. Contrairement à nos anticipations, notre étude ne démontre pas de conflits systématiques causés par la coexistence du système de relations du travail et de la responsabilité fiduciaire des régimes complémentaires de retraite. Les enjeux identifiés ne sont pas perçus comme des sources de tensions, mais relèvent plutôt de la confusion des rôles. La qualité de la gouvernance des régimes de retraite dépend des ressources investies par les acteurs des relations du travail dans l'accompagnement des membres des comités de retraite.
\end{abstract}

MOTS-CLÉs: Québec, représentation collective, gouvernance, régime de retraite, responsabilité fiduciaire, conflit de rôle.

\section{Introduction}

Cet article s'intéresse aux conflits de rôles potentiels dans la gouvernance des régimes complémentaires de retraite au Québec. Nous exploitons une particularité de la législation québécoise, soit l'obligation de créer un comité de retraite,

Daniel Coulombe, professeur, École de comptabilité, Université Laval (daniel.coulombe@rlt.ulaval.ca).

Esther Déom, professeure, Département des relations industrielles, Université Laval (esther.deom@rlt.ulaval.ca). Frédéric Hanin, professeur, Département des relations industrielles, Université Laval (frederic.hanin@rlt.ulaval.ca). Annette Hayden, professionnelle de recherche, Université Laval (annette.hayden@rlt.ulaval.ca).

Les auteurs ont choisi de placer leurs noms en ordre alphabétique.

Cette recherche a bénéficié du financement de la Chaire sur la Gouvernance de I'Université Laval et du support de Retraite Québec pour l'accès aux personnes-ressources des comités de retraite. Nous tenons à remercier les évaluateurs de la revue, les participants aux ateliers lors des colloques du CRIMT 2010 tenu à I'Université Laval, du CRIMT 2012 tenu aux HEC Montréal, ainsi que lors du séminaire 2014 de la Chaire sur la Gouvernance de I'Université Laval, pour leurs questions et leurs commentaires. Les erreurs et omissions qui pourraient demeurer sont de la responsabilité des auteurs. 
sur lequel siègent des membres représentant l'employeur, les syndicats, les travailleurs, les retraités ainsi que des membres indépendants. La création d'une structure de gestion composée de personnes qui ne sont pas toutes nécessairement formées pour agir à titre de fiduciaire, ou qui n'ont pas nécessairement les connaissances suffisantes pour s'acquitter de cette tâche, crée un climat propice aux conflits de rôles entre l'intérêt des parties prenantes aux relations du travail et la responsabilité fiduciaire qui concerne le bien-être des participants au régime.

Le Québec présente plusieurs particularités qui ont une influence sur la gouvernance des régimes de retraite. Une première particularité, par rapport à d'autres provinces au Canada, est d'avoir institutionnalisé la responsabilité fiduciaire sous la forme d'un comité de retraite, dont l'ensemble des membres a une responsabilité fiduciaire. Une seconde particularité vient du vieillissement plus rapide de sa population, comparativement à d'autres provinces, ce qui accentue les enjeux financiers (montant des fonds accumulés pour verser des rentes viagères) et de gouvernance des régimes complémentaires de retraite, notamment ceux à prestations déterminées dans lesquels l'évaluation actuarielle est faite sur la base de taux de mortalité anticipés (Québec, 2013). Une troisième particularité provient du taux de présence syndicale plus élevé au Québec que dans le reste du Canada (39,6\% contre $29,4 \%)$, notamment dans le secteur public et para-public, ou encore dans le secteur de la construction (Labrosse, 2016). La présence syndicale a été un facteur déterminant pour la mise en place de régimes complémentaires de retraite.

Nous définissons les conflits de rôle dans la gouvernance des régimes de retraite à partir de la coexistence du système des relations du travail et de la responsabilité fiduciaire des régimes complémentaires de retraite. De plus, les mêmes personnes assument souvent les deux fonctions de représentation des parties et de membre de comité de retraite. La notion de conflit de rôles diffère de la notion de conflit d'intérêts, dans la mesure où le premier s'avère être une caractéristique structurelle, alors que le second porte sur des intérêts personnels (Royal et Brassard, 2010; Biddle, 1979). En cas de conflit d'intérêts, par exemple, il est d'usage que la personne ne prenne pas part à une décision, car cela la placerait dans une situation difficile. Ce n'est pas le cas dans un conflit de rôle.

À partir de ces arguments, nous posons les questions de recherche suivantes:

- Quelles sont les sources de conflits de rôles perçus par les membres de comité de retraite?

- Les enjeux de financement des régimes de retraite favorisent-ils la présence de conflits de rôle?

- La gouvernance des régimes de retraite est-elle une source de tension?

Pour répondre à ces questions de recherche, nous avons choisi une démarche 
basée sur une méthodologie mixte qui a consisté à envoyer un questionnaire à des membres de comités de retraite et à réaliser, par la suite, des entrevues individuelles avec certains d'entre eux afin d'approfondir la compréhension de leur situation, ainsi que leurs réponses aux questions. Nous avons pu ainsi mesurer l'étendue des situations potentiellement conflictuelles et comprendre la nature des conflits ou leur absence.

La première section décrit le cadre institutionnel de la gouvernance des régimes complémentaires de retraite au Québec. La seconde section présente le cadre théorique d'analyse des conflits de rôle dans les régimes de retraite. La troisième section porte sur la méthodologie de recherche et les liens entre la dimension quantitative et la dimension qualitative. La quatrième section analyse les résultats de recherche concernant les relations du travail et les enjeux dans le domaine de la gouvernance. La dernière section discute des résultats obtenus et des prolongements de l'enquête.

\section{Cadre institutionnel de la gouvernance des régimes complémentaires de retraite au Québec}

Cette section présente les grandes lignes du système de revenus de retraite au Québec, particulièrement les régimes complémentaires de retraite qui sont I'objet de notre étude.

\section{La structure du système de revenus de retraite au Québec}

Le fonctionnement de la retraite au Canada et au Québec se décline en 3 paliers (voir encadré 1).

\section{ENCADRÉ 1}

Le système de revenu de retraite au Québec

\section{Niveaux}

1. Pension de sécurité de la vieillesse (PSV) et supplément de revenu garanti (SRG): répartition

2. Régime des rentes du Québec (RRQ): capitalisation et répartition

\section{Prestations}

Prestations universelles versées à 65 ans

\section{Financement}

Budget fédéral

Cotisations de l'employeur et de l'employé obligatoire jusqu'au maximum de gains admissibles

- Cotisations fiscalement déductibles

3. Régimes de pension agrées complémentaires (capitalisation)
Rente normale : vise à couvrir 25 pour cent du salaire pendant maximum des gains admissibles

- Prestations définies
- Cotisations définies
- Hybrides
- Prestations cibles
- REER (individuels ou collectifs)

- Prestations définies

Cotisations définies

Prestations cibles
- Cotisations de l'employeur et de l'employé (obligatoires ou facultatives) qui peuvent être négociées collectivement

- Cotisations fiscalement déductibles 
Au premier palier se trouve le régime de base de la sécurité de la vieillesse, sous la responsabilité du gouvernement fédéral. Ce palier fournit une prestation minimale. Le deuxième palier, qui relève du gouvernement du Québec, constitue un régime de retraite obligatoire pour tous les travailleurs. II s'agit du Régime de rentes du Québec (RRQ)1, qui vient compléter le premier palier fédéral. On retrouve au troisième palier les régimes complémentaires de retraite établis par les employeurs, et négociés avec les syndicats ou les travailleurs. C'est sur ce dernier volet que porte notre recherche sur les conflits de rôle. Le régime de retraite complémentaire constitue un contrat entre l'employeur et les participants. Dans le cas d'un régime à prestations déterminées (ci-après régime $P D)$, les travailleurs ont la promesse d'une rente selon certains paramètres, par exemple, $2 \%$ du salaire moyen des 5 meilleures années par année de service. ${ }^{2}$ Dans le cas d'un régime à cotisations déterminées (ci-après régime $C D$ ), aucune promesse n'est faite aux participants pour un montant de rente à la retraite; toutefois, les cotisations sont accumulées et gérées collectivement dans une caisse de retraite. Dans ce type de régime, le participant quitte avec la valeur accumulée des cotisations et des rendements obtenus au cours de sa carrière ${ }^{3}$.

\section{Les régimes complémentaires de retraite et la responsabilité fiduciaire}

Trois éléments peuvent être identifiées pour expliquer I'utilisation de la structure des fiducies dans le cas des régimes de retraite (Clark, Caerlewy-Smith et Marshall, 2007; McGill, 2005; Hawley, 2014). Tout d'abord, la fiducie vise exclusivement au bien-être des bénéficiaires dans une perspective de long terme. Ensuite, la fiducie dissocie les parties au contrat de retraite (I'employeur et chacun des employés couverts) de l'administrateur du régime (le comité de retraite), ce qui peut, dans certains cas, éviter la présence de conflits d'intérêt dans l'utilisation des fonds, surtout quand ils ont un caractère collectif. En même temps, cela signifie que la gestion est déléguée à l'administrateur par le propriétaire, mais que ce dernier demeure responsable des obligations vis-à-vis des bénéficiaires. Enfin, la fiducie repose sur la reddition de compte de l'administrateur de la fiducie, même si cette responsabilité repose uniquement sur les moyens mis en œuvre et non sur les résultats financiers. L'obligation de moyen implique souvent I'utilisation de la gestion déléguée à travers des mandats confiés à des gestionnaires de fonds spécialisés, ainsi que le recours à des fournisseurs de services (évaluation actuarielle, membres externes de comités de retraite, avis juridiques, etc.).

La Loi sur les régimes complémentaires de retraite (L.R.Q., chapitre R-15.1, Loi $R C R$ par la suite) exige la création d'un comité de retraite pour chaque régime de retraite de plus de 25 participants sous sa juridiction qu'il soit à cotisations déterminées, à prestations déterminées ou à prestations cibles. 
C'est une particularité unique au Québec. Ce comité est, en fait, l'équivalent d'un conseil d'administration pour les régimes. La loi exige que le comité ait au moins un membre nommé parmi les participants actifs, un membre nommé parmi les participants inactifs (retraités) et un membre indépendant. Toutefois, les parties prenantes aux régimes de retraite ajoutent généralement des membres à leur comité, nommés par l'employeur, par le syndicat et par les participants. II n'y a pas de normes sur la taille de ce comité et il peut être paritaire ou non. La gouvernance des régimes de retraite comporte ainsi une double dimension collective, à la fois, par la représentation au sein du comité de retraite du promoteur du régime (en général, il s'agit de l'employeur) des groupes de participants (participants actifs, bénéficiaires retraités ou conjoints), mais également par la représentation des parties impliquées dans les relations du travail (employeur et syndicat de travailleurs). L'analyse de la gouvernance est donc différente de celle d'une compagnie avec des actionnaires.

La Loi $R C R$ impose une responsabilité fiduciaire à l'administrateur du régime, soit le comité de retraite et ses membres. Le fiduciaire doit gérer les actifs qui lui sont confiés en respectant des règles et une éthique très strictes. Notamment, le fiduciaire doit agir avec honnêteté et loyauté dans l'intérêt exclusif des déposants, comme le ferait en pareilles circonstances une personne prudente, et en utilisant toutes les informations et compétences professionnelles qu'elle possède. Le comité doit administrer le régime, notamment s'assurer que les cotisations sont dûment versées à la caisse, déterminer et verser les prestations de retraite, gérer et investir les actifs de la caisse, faire préparer les évaluations actuarielles et effectuer toutes les communications requises. Les membres de ces comités n'ont pas d'obligation légale de détenir toutes les compétences et la formation pour assumer ces tâches, mais la loi permet, en contrepartie, au comité de s'appuyer sur des experts pour les aider dans leur travail.

Le régime de retraite fait partie de la rémunération globale des travailleurs. En conséquence, le financement et l'évolution du régime dépendent généralement d'accords collectifs entre les acteurs des relations du travail, soit directement à travers l'inscription du régime de retraite dans la convention collective, soit indirectement par l'action législative ou encore la comparaison des conditions de travail entre entreprises ou groupes professionnels d'une même entreprise.

\section{Cadre d'analyse des conflits de rôle}

Plusieurs études internationales ont porté sur la question des conflits d'intérêt ou des conflits de rôle dans la gouvernance des régimes de retraite, soit celui des tensions que peuvent créer, chez les membres de comités de retraite, la confusion entre le rôle de fiduciaire du régime de retraite et le rôle de représentant nommé ou désigné par l'employeur, le syndicat ou les retraités (Cocco et Volpin, 2007; 
Kakabadse, Kakabadse et Kouzmin, 2003). D'autres études se sont penchées sur la différence entre les conflits de rôles et conflit d'intérêts (Royal et Brassard, 2010; Biddle, 1979).

La figure 1 présente le cadre d'analyse des conflits de rôle dans les régimes de retraite.

\section{FIGURE 1}

Le cadre d'analyse des conflits de rôle dans les régimes complémentaires de retraite

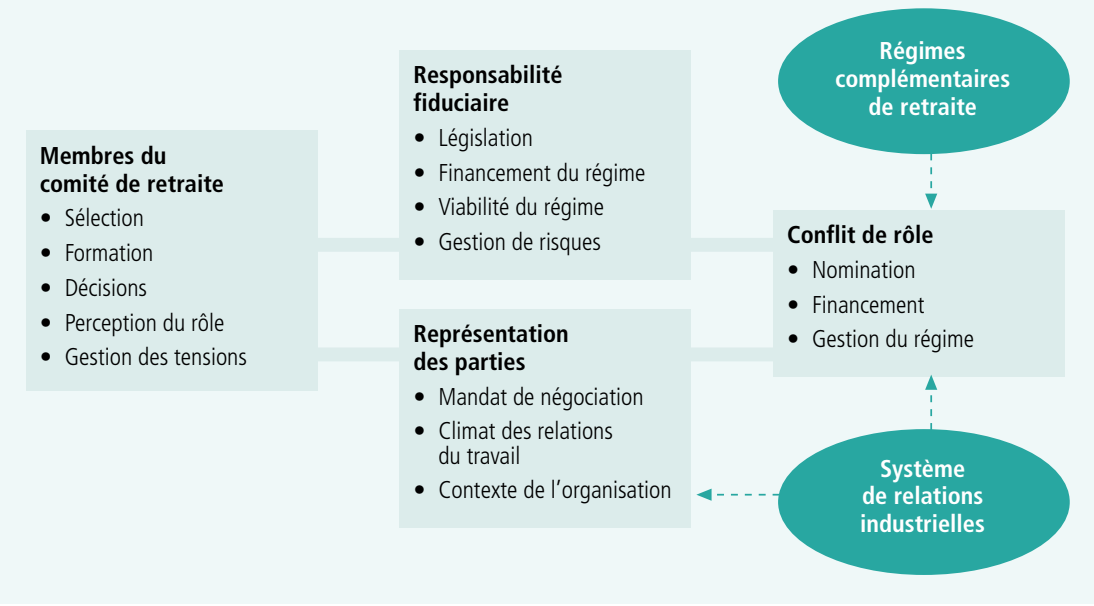

L'hypothèse testée dans cette recherche est la suivante: la présence de conflits de rôles provient de la coexistence du système des relations du travail et du système prévu par la Loi $R C R$ sur les régimes complémentaires de retraite. En effet, les mêmes personnes assument souvent les deux fonctions de représentation des parties et de membre de comité de retraite et elles fonctionnent ainsi à l'intérieur de deux régimes aux règles particulières.

En théorie, il semble facile d'établir une démarcation claire entre ces deux rôles (fiduciaire et de représentant des parties) puisqu'ils s'exercent à des niveaux différents, dans des lieux différents et portent sur des sujets différents. Notre recherche permet d'aller vérifier ce qu'il en est en pratique. Ainsi, pour les raisons évoquées précédemment, nous faisons I'hypothèse que les tensions risquent d'être exacerbées en contexte syndiqué (Carmichael, 2005; Hebb Fung et Rogers, 2001; McCarthy, 2014).

\section{Les conflits de rôle dans la nomination au comité de retraite}

Le conflit de rôle dans la représentation au comité de retraite peut survenir lorsque les membres ne font pas la distinction entre l'intérêt d'une partie ou 
d'un groupe dont ils ont reçu un mandat (employeur, syndicat, association de retraités) et la responsabilité fiduciaire qui concerne l'administration du régime de retraite dans son ensemble. L'existence d'un conflit de rôle n'est cependant pas indépendante du contexte légal qui détermine les frontières de la responsabilité fiduciaire.

Selon la $L o i R C R$, les parties syndicales et patronales nomment souvent des représentants au comité de retraite. De toute évidence, la possibilité de conflit de rôles est exacerbée par le nombre de nomination des parties sur le comité. En 1996, il y avait près de 10000 membres de comités de retraite au Québec (Lizée, 2002). À partir de notre enquête par questionnaire et en extrapolant les résultats obtenus pour 202 régimes sur les 780 régimes sous la surveillance de Retraite Québec, il y aurait environ 7400 membres de comités de retraite au Québec en 20144', alors que l'obligation minimum légale serait de l'ordre de 2300 membres.

\section{Les conflits de rôle dans le financement des régimes de retraite}

À l'origine, la Loi $R C R$ était fondée sur un modèle-type d'un régime de retraite mis en place par un promoteur souvent unique (un employeur) qui pouvait administrer ou même mettre fin au régime de sa propre initiative, dans la mesure où il supportait seul l'ensemble des risques. Les régimes de retraite sont tenus d'adopter une politique de gestion des risques et une politique de placement, mais la politique de financement du régime n'était pas obligatoire avant l'adoption, le 26 novembre 2015, de la Loi modifiant la Loi sur les régimes complémentaires de retraite principalement quant au financement des régimes de retraite à prestations déterminées (2015, chap. 29). Le financement était traditionnellement du ressort de l'employeur qui supportait seul les risques en la matière (surplus, déficits). Lors de notre enquête, $54 \%$ des régimes n'avaient pas de politique de financement. Par ailleurs, $71,4 \%$ des répondants indiquaient que le régime était négocié collectivement.

Les récentes modifications législatives pour les régimes des secteurs municipal, privé et universitaire ont introduit davantage de partage des risques, le partage des risques entre le promoteur et les participants tendant maintenant à remplacer I'ancien modèle, ce qui peut engendrer une autre source de conflit de rôle pour les membres du comité de retraite. En effet, le partage du financement du régime de retraite est souvent déterminé dans le cadre de la négociation de la convention collective alors que le coût du régime de retraite est déterminé par le comité de retraite au moment de l'évaluation actuarielle. Dans la $L o i R C R$, il existe une possibilité pour le comité de retraite de faire en tout temps des recommandations aux parties qui ont le pouvoir de modifier le régime de retraite ( $L o i R C R$, art. 150.1).

Des conflits de rôle peuvent, en effet, apparaitre dès lors que le contexte de la négociation collective entre en tension avec les besoins de financement du 
régime de retraite. On peut même en retrouver en situation de surplus du régime de retraite, par exemple, lorsque les parties à la négociation collective souhaitent utiliser les surplus afin de diminuer, à court terme, le financement du régime, au détriment de sa viabilité à long terme.

La gestion du financement du régime de retraite est donc de plus en plus partagée entre le promoteur et les participants, mais sans autre encadrement obligatoire que la convention collective lorsque les participants actifs sont représentés par un syndicat. II peut alors exister un conflit entre les objectifs de la négociation collective et le financement du régime de retraite.

\section{Les conflits de rôle associés aux délégations de gestion dans les régimes de retraite}

En raison du mode de représentation dans les comités de retraite, l'expertise n'est pas toujours là, mais la délégation est possible.

En effet, l'article 152 de la Loi $R C R$ permet les délégations de pouvoir. On retrouve ainsi plusieurs formes de délégation:

- de l'administration du régime à l'employeur ou à une institution financière qui perçoit les cotisations et verse les prestations aux bénéficiaires;

- de gestion de la politique de placement à des comités de placement qui sont des sous-comités du comité de retraite ou encore à des institutions financières qui offrent des «formules» de placement directement aux participants, comme dans la plupart des régimes à cotisations déterminées et des REER collectifs.

De plus, l'article 151.2 de la Loi $R C R$ prévoit l'établissement d'un règlement intérieur régissant le fonctionnement et la gouvernance du comité de retraite. En l'absence de négociation avec l'autre partie, le promoteur (l'employeur) décide seul des conditions d'établissement du régime de retraite, notamment les conditions de sa modification (alinéa 15), les conditions d'utilisation des excédents (alinéas 16 et 17) et la composition du comité de retraite (alinéa 2). Ce rapport de force en faveur de l'employeur rend difficile l'équilibre entre les parties dans l'administration du régime, dès lors que c'est le comité de retraite dans son ensemble qui porte la responsabilité fiduciaire.

Cette délégation peut être source de conflit, comme par exemple au niveau du choix du délégataire. Une délégation à l'employeur peut être mal perçue par les membres syndicaux qui peuvent y voir un conflit de rôle. Le choix de l'actuaire est aussi déterminant dans l'établissement du coût du régime et sa nomination peut être une source de conflit.

En conséquence, dans la structure institutionnelle actuelle de la gouvernance des régimes de retraite au Québec, les sources potentielles de conflits de rôle 
sont multiples et concernent surtout les conflits entre la représentation des intérêts d'une des parties associées à la négociation collective et la responsabilité fiduciaire. L'enquête sur les conflits de rôle permet ainsi d'analyser plus en détail les sources et la nature des conflits du point de vue des membres de comités de retraite au Québec.

\section{Méthodologie de l'enquête}

Tel que mentionné précédemment, nous avons envoyé un questionnaire à des membres de comités de retraite et avons, par la suite, réalisé des entrevues individuelles avec certains d'entre eux afin d'approfondir la compréhension de leur situation ainsi que des réponses aux questions.

Pour constituer notre échantillon, nous avons procédé en plusieurs étapes, qui sont décrites dans la figure 2 .

\section{FIGURE 2}

Processus de recrutement par questionnaire

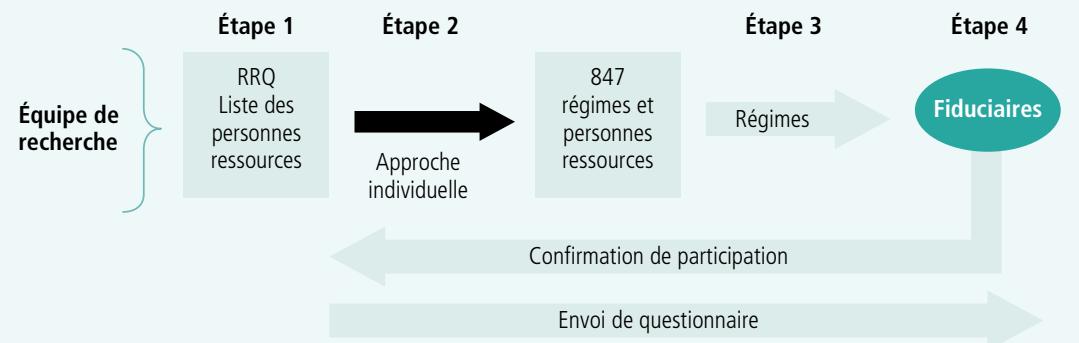

Étape 5

Dans une première étape, nous avons obtenu de Retraite Québec les coordonnées des personnes-ressources de tous les régimes de retraite sous leur juridiction, soit 847 régimes. Nous avons, ensuite, contacté individuellement chaque personneressource pour lui demander de transmettre à chaque membre de son comité de retraite une demande les invitant à répondre à notre questionnaire. Comme nous ne connaissions pas l'identité des membres des comités de retraite, il est difficile de savoir combien de membres de comités ont été réellement rejoints, mais on en estime le nombre potentiel à près de 7400 (une même personne peut être membre de plusieurs comités de retraite). Les personnes intéressées nous contactaient et nous leur faisions parvenir le questionnaire par envoi électronique ou postal. Le questionnaire pouvait être rempli en format papier, PDF ou Web. Au total, nous avons reçu 204 questionnaires complétés.

Les caractéristiques des répondants au questionnaire sont présentées dans le tableau 1. 
TABLEAU 1

Caractéristiques des répondants de l'enquête (en \%)

\begin{tabular}{|c|c|c|c|c|c|c|c|c|c|c|c|}
\hline \multicolumn{2}{|c|}{ Mode de désignation } & \multicolumn{2}{|c|}{$\begin{array}{l}\text { Ancienneté comme } \\
\text { membre du comité }\end{array}$} & \multicolumn{2}{|c|}{ Genre } & \multicolumn{2}{|c|}{ Âge } & \multicolumn{2}{|c|}{ Type d'emploi occupé } & \multicolumn{2}{|c|}{ Formation reçue } \\
\hline Syndicat & 14,36 & $<1$ an & 4,39 & Homme & 80,0 & $\begin{array}{r}\text { Moins } \\
\text { de } 50 \text { ans }\end{array}$ & 36,5 & Col bleu & 6,99 & $\begin{array}{l}\text { Relations } \\
\text { du travail }\end{array}$ & 58,54 \\
\hline Employeur & 44,55 & 1 à 2 ans & 8,29 & Femme & 20,0 & $\begin{array}{r}50- \\
60 \text { ans }\end{array}$ & 47,5 & Col blanc & 28,50 & $\begin{array}{r}\text { Finance, } \\
\text { comptabilité, } \\
\text { actuariat }\end{array}$ & 65,85 \\
\hline Indépendant & 8,91 & $\begin{array}{l}\text { Plus de } \\
2 \text { ans }\end{array}$ & 87,32 & & & $\begin{array}{l}\text { Plus de } \\
60 \text { ans }\end{array}$ & 16,0 & Cadre & 41,94 & $\begin{array}{r}\text { Ressources } \\
\text { humaines }\end{array}$ & 73,40 \\
\hline Participants actifs & 21,78 & & & & & & & Professionnels & 22,58 & & \\
\hline Participants inactifs & 10,40 & & & & & & & & & & \\
\hline
\end{tabular}

Note: Les chiffres sont indiqués en pourcentage de l'échantillon. La question sur la formation reçue pouvait comporter plus d'une réponse.

\section{TABLEAU 2}

Caractéristiques des régimes de retraite (en \%)

\begin{tabular}{|c|c|c|c|c|c|c|c|c|c|}
\hline \multicolumn{2}{|c|}{ Secteurs } & \multicolumn{2}{|c|}{ Types de régime } & \multicolumn{2}{|c|}{$\begin{array}{l}\text { Nombre de participants } \\
\text { actifs et inactifs }\end{array}$} & \multicolumn{2}{|c|}{$\begin{array}{c}\text { Présence de } \\
\text { plusieurs régimes }\end{array}$} & \multicolumn{2}{|c|}{$\begin{array}{l}\text { Taille du comité } \\
\text { de retraite }\end{array}$} \\
\hline Parapublic & 45,18 & PD & 64,18 & $>2500$ & 3,5 & Oui & 27,32 & $>15$ personnes & 6,90 \\
\hline Fabrication & 31,47 & $C D$ & 26,37 & $1000-2500$ & 15,5 & Non & 71,04 & 10-15 personnes & 36,95 \\
\hline Services & 16,24 & $\mathrm{H}$ & 8,46 & $500-1000$ & 11,5 & Ne sais pas & 1,64 & 6-9 personnes & 49,26 \\
\hline Primaire & 7,11 & $P C$ & 1,00 & $<500$ & 68,5 & & & 3-5 personnes & 6,90 \\
\hline
\end{tabular}

Note: Résultats indiqués en pourcentage de l'échantillon. PD : Prestations déterminées; $\mathrm{CD}$ : Cotisations déterminées; H :Hybride; PC: prestations cibles 
Le tableau 2 présente les caractéristiques des régimes de retraite retenus dans I'enquête par questionnaire. On y apprend ainsi que la majorité des régimes représentés sont des régimes à prestations déterminées. II y a également une forte proportion de régimes avec moins de 500 participants 5 .

Finalement, 94 répondants ont souhaité nous rencontrer en entrevue individuelle. Nous avons dû faire une sélection basée, notamment, sur l'appartenance sectorielle, la représentation (employeurs, participants, membres externes), la répartition géographique et le type de régime de retraite. Les entrevues se sont déroulées entre le $1^{\text {er }}$ décembre 2013 et le 15 septembre 2014.

Les caractéristiques des répondants aux entrevues sont présentées dans le tableau 3.

\section{TABLEAU 3}

Caractéristiques des répondants aux entrevues

\begin{tabular}{|c|c|c|c|c|c|}
\hline Numéro & Secteur & $\begin{array}{l}\text { Type de } \\
\text { régime }\end{array}$ & $\begin{array}{l}\text { Taille du } \\
\text { régime }\end{array}$ & Représentant & $\begin{array}{l}\text { Nombre de } \\
\text { régimes }\end{array}$ \\
\hline 1 & Universitaire & PD & $1000-2500$ & Retraités & 1 \\
\hline 2 & Universitaire & $P D$ & $>2500$ & Employeur & 4 \\
\hline 3 & $\begin{array}{l}\text { Universitaire- } \\
\text { Municipal }\end{array}$ & $P D$ & $>2500$ & $\begin{array}{l}\text { Administrateur- } \\
\text { Externe }\end{array}$ & 7 \\
\hline 4 & Privé & $P D-P C$ & $>2500$ & Employeur & 17 \\
\hline 5 & Universitaire & $P D-C D$ & $1000-2500$ & Externe & 2 \\
\hline 6 & Privé & PD & $>2500$ & Retraité-Externe & 4 \\
\hline 7 & Privé & $C D$ & $500-1000$ & Syndicat & 1 \\
\hline 8 & Universitaire & PD & $>2500$ & Administrateur & 1 \\
\hline 9 & Privé & $\mathrm{H}$ & $<500$ & Employeur & 2 \\
\hline 10 & Universitaire & PD & $1000-2500$ & Syndicat & 1 \\
\hline 11 & Universitaire & PD & $1000-2500$ & Syndicat & 1 \\
\hline 12 & Privé & PD & $1000-2500$ & Employeur & 1 \\
\hline 13 & Municipal & PD & $<500$ & Syndicat & 1 \\
\hline 14 & Privé & $C D$ & $<500$ & Syndicat & 1 \\
\hline 15 & Municipal & PD & $500-1000$ & Syndicat & 1 \\
\hline 16 & Municipal & PD & $1000-2500$ & Employeur & 2 \\
\hline 17 & Privé & $C D$ & $<500$ & Syndicat & 1 \\
\hline 18 & Municipal & PD & $1000-2500$ & Retraité & 1 \\
\hline
\end{tabular}

Notes : PD : Prestations déterminées; CD : Cotisations déterminées; $\mathrm{H}$ : Hybride; PC : prestations cibles.

Nous avons, ainsi, effectué 18 entrevues semi-dirigées de $2 \mathrm{~h}$ en moyenne, ce qui rejoignait un total de 49 régimes. Trois personnes représentaient les retraités, cinq personnes représentaient l'employeur, sept personnes représentaient un syndicat d'employés et trois personnes avaient été nommées en tant que membres externes ${ }^{6}$. 
Le questionnaire comprenait cinq sections, alors que les entrevues reprenaient, en les approfondissant, les thématiques abordées dans le questionnaire:

1. description de l'entreprise (secteur d'activités, taille);

2. caractéristiques du régime (taille, participants, type de régime);

3. fonctionnement du comité de retraite;

4. caractéristiques du répondant (sexe, type d'emploi, etc.);

5. identification des personnes intéressées à être interrogées en entrevue.

L'analyse statistique des réponses aux questionnaires s'est faite par traitements univariés et multivariés avec le logiciel SAS. Les entrevues ont été réalisées en fonction des réponses au questionnaire de chacune des personnes interrogées. Les entrevues portaient sur le fonctionnement du comité de retraite, les situations potentielles de conflits de rôle, et les sources de tensions dans la gouvernance des régimes de retraite. Les comptes rendus des entrevues ont fait, ensuite, l'objet d'une analyse de contenu thématique afin d'identifier la dynamique des relations au sein du comité de retraite, la dynamique des rapports collectifs du travail, et les enjeux sur les ressources à la disposition des membres des comités de retraite dans le cadre de l'administration du régime dans le contexte de la Loi $R C R$.

\section{Résultats}

Cette section décrit les résultats de l'étude. Nous abordons les résultats concernant les sources de conflits de rôles évoquées par les participants, puis, plus spécifiquement, les résultats relatifs aux enjeux liés à l'intégration des membres, au financement des régimes, à la gouvernance des régimes et au cas particulier des régimes $C D$.

\section{Les sources de conflits de rôles}

Nous avons d'abord demandé directement aux répondants s'il y avait des sources de tension au sein de leur comité de retraite. Les résultats sont présentés dans le tableau 4.

\section{TABLEAU 4}

Sources de tensions (en \%)

Problèmes ou tensions identifiées

Fréquence

Aucune tension 28,80

\begin{tabular}{ll}
\hline Tensions reliées aux relations du travail & 4,88
\end{tabular}

\begin{tabular}{lr}
\hline Tensions avec les fournisseurs de service & 7,30
\end{tabular}

\begin{tabular}{ll}
\hline Tensions au niveau des aspects financiers du régime & 50,20
\end{tabular}

\begin{tabular}{ll}
\hline Tensions internes, viabilité du régime, gouvernance & 28,80
\end{tabular}

\begin{tabular}{ll}
\hline Autres & 7,32
\end{tabular}

Note: Résultats indiqués en pourcentage de l'échantillon. Les répondants pouvaient indiquer plusieurs réponses si une tension était mentionnée. 
Nous constatons qu'il n'existait aucune source de tension pour 28,8\% des répondants. De plus, une majorité de tensions soulevées par les répondants, le cas échéant, n'était pas associée directement à la responsabilité fiduciaire, mais plutôt à des considérations des parties syndicales et patronales. Par exemple, les tensions soulevées par les aspects financiers relèvent de la responsabilité des parties et non du comité de retraite. Par ailleurs, nous remarquons que les conflits de rôle sont davantage présents dans les régimes comportant plusieurs catégories d'emploi, les entrevues ayant aussi confirmé ce résultat.

Pour compléter cette première analyse, nous avons demandé aux répondants s'il y avait des sujets importants ou prioritaires identifiés par le comité pour la prochaine année d'exercice. Cette question visait à identifier indirectement les sources de tensions potentielles. Pour nous permettre de faire des liens avec la qualité des relations du travail, nous les avons aussi questionnés afin d'avoir leur perception de la qualité des relations du travail. Ces résultats apparaissent au tableau 5.

TABLEAU 5

Enjeux des régimes de retraite et sources de tensions (en \%)

Sujets importants dans l'année à venir

Qualité des relations du travail

Gouvernance du régime (politique de placement,

\begin{tabular}{lcll} 
sélection membres, risques financiers) & 37 & Très difficiles & 1,51 \\
\hline Viabilité du régime & 27 & Plutôt difficiles & 13,07 \\
\hline Financement du régime & 29 & Plutôt bonnes & 61,81 \\
\hline Relation avec l'employeur & 5 & Très bonnes & 23,62 \\
\hline
\end{tabular}

Note: Résultats indiqués en pourcentage de l'échantillon. Les répondants pouvaient indiquer plusieurs réponses.

Environ $37 \%$ des répondants ont indiqué des enjeux reliés à leur rôle de fiduciaire, soit la gouvernance du régime. La plupart des autres considérations soulevées ici ne sont pas liées à la gouvernance des régimes de retraite, mais aux relations du travail. C'est ainsi le cas pour la viabilité du régime ( $27 \%$ des répondants), le financement du régime ( $29 \%$ des répondants) ou des relations avec l'employeur (5\% des répondants). La qualité des relations du travail a peu à voir avec ces résultats, sauf pour les enjeux de viabilité du régime. Les participants jugeant les relations du travail moins favorables sont plus susceptibles de déclarer des enjeux reliés à la viabilité du régime.

On peut donc présumer, pour l'identification des enjeux et tensions, qu'il y a une confusion des rôles entre la représentation des parties et la responsabilité fiduciaire de la part de membres qui assurent souvent les deux rôles en même temps dans les organisations. 


\section{Les sources de tensions dans la nomination et l'intégration des membres de comité}

Bien que quelques participants ont déclaré que leur nomination au sein du comité de retraite avait généré des tensions, pour la très grande majorité (soit $95 \%$ leur nomination s'est effectuée sans heurt. Les participants aux entrevues ont confirmé l'absence de conflit explicite au sein des comités de retraite.

Au niveau de l'intégration, les répondants soulignent la nécessité d'une double formation, à la fois dans le domaine de la finance, et dans le domaine des relations industrielles. II a souvent été mentionné dans les entrevues que la période jugée adéquate de formation pouvait aller jusqu'à 5 ans pour les régimes de retraite de grande taille. Les répondants ont toujours fait référence, soit à une expérience professionnelle préalable dans le domaine des régimes de retraite, soit à des dispositifs de libération dans leur emploi, pour être en mesure d'assumer pleinement leurs responsabilités.

Selon les participants, il est difficile pour une même personne d'être à la fois partie prenante des négociations collectives et membre du comité de retraite dans les régimes de petite taille, dès lors qu'un arbitrage existe entre les demandes salariales et le financement du régime de retraite. Pourtant, la mise en place d'un régime et son évolution est souvent le résultat d'initiatives syndicales à l'occasion de la négociation collective. La négociation collective est d'autant plus importante que le milieu de travail connaît des restructurations ou que le régime de retraite rencontre des problèmes de financement ou doit évoluer à la suite de modifications législatives.

L'engagement des parties est également bénéfique pour la qualité de la gouvernance des régimes de retraite. C'est le cas lorsque les parties soutiennent la formation initiale et continue des membres du comité de retraite, financent la participation des membres du comité de retraite grâce à des libérations, offrent du soutien technique (actuariel, juridique, relations du travail), comme dans le cas des centrales syndicales ou des grandes entreprises. C'est donc le niveau de ressources « investies» par les parties qui est souvent déterminant pour assurer la qualité de la gouvernance des régimes de retraite.

La qualité de la délibération collective dépend également de l'intérêt des participants vis-à-vis de questions qui apparaissent souvent comme trop techniques et lointaines. Plusieurs comités fonctionnent avec des postes vacants (18\%), en grande majorité des régimes à prestations déterminées. Plusieurs répondants aux entrevues ont mentionné que les comités de retraite doivent alors faire preuve d'imagination afin de favoriser la participation aux assemblées annuelles du régime de retraite. Cependant, les décisions importantes concernant l'évolution de la structure du régime de retraite seront abordées dans les assemblées syndi- 
cales et au moment de la négociation de la convention collective. Le contenu des entrevues nous amène à penser qu'il faudrait peut-être modifier la Loi $R C R$ pour permettre d'autres modalités de communication entre les membres du comité de retraite et les participants.

\section{Les enjeux de financement dans les comités de retraite}

L'enjeu du financement du régime, aussi bien pour le financement du service courant que pour les déficits, est la principale source potentielle de tensions au sein du comité de retraite.

Ces tensions peuvent s'expliquer, notamment, par l'absence de politique de financement obligatoire pour les régimes de retraite. Ainsi, 39\% seulement des répondants ont déclaré avoir une politique de financement. Traditionnellement, le financement du régime était assuré en grande partie par le promoteur, c'est-à-dire l'employeur. Avec l'évolution du financement à coût partagé pour le service courant, et maintenant du déficit suite à des modifications législatives, les problèmes de financement des régimes de retraite sont susceptibles de s'ajouter aux tensions inhérentes aux relations du travail dans le cadre de la négociation des conventions collectives. Nos résultats indiquent que la présence d'une politique de financement n'est pas une dimension significative pour expliquer l'absence de considérations et de tensions associées au financement du régime de retraite. Cet enjeu dépasse visiblement la question de la qualité de la gouvernance du comité de retraite, ainsi que les moyens individuels nécessaires au respect de la responsabilité fiduciaire.

Les entrevues ont montré que les sources de tension reliées au financement sont généralement gérées à l'extérieur des réunions du comité de retraite par des sous-comités ou lors de rencontres informelles. Les tensions ont le plus de chance de survenir lors des évaluations actuarielles, lorsqu'il faut déterminer des hypothèses qui auront des impacts sur les cotisations pour le service courant, ainsi que sur le niveau du déficit (de solvabilité et de capitalisation).

\section{Les enjeux liés à la gouvernance des régimes}

Les éléments de gouvernance qui ont été identifiés par la recherche concernent autant les régimes à prestations déterminées que les régimes à cotisations déterminées. La délégation, le fonctionnement de comité, les moyens mis à la disposition des membres de comité de retraite sont les enjeux les plus souvent mentionnés lors des entrevues.

Selon les données de notre enquête, l'administration a, ainsi, été totalement ou en partie déléguée à l'employeur dans une large proportion des régimes. De plus, cette délégation à l'employeur est majoritairement présente lorsque les répondants déclarent des relations du travail difficiles. L'employeur bénéficie 
de ressources appropriées pour assurer adéquatement la gestion du régime, mais nos entrevues démontrent qu'une telle délégation est souvent perçue négativement par les représentants des participants. $61 \%$ des répondants ont indiqué que la politique de placement était déléguée à des gestionnaires externes et près de $50 \%$ des répondants considèrent que les membres de leur comité de retraite n'ont pas une formation adéquate en matière de placement. Nos résultats démontrent que cette délégation se fait significativement plus souvent pour les plus petits régimes. Les régimes de retraite qui ont moins de 1000 participants, ainsi que les régimes à cotisations déterminées, ont une probabilité plus forte que la moyenne de déléguer une partie des responsabilités à des fournisseurs externes au régime de retraite. Les régimes qui comptent plus de 1000 participants recourent davantage à des sous-comités du comité de retraite afin de répartir les responsabilités.

Les entrevues ont indiqué que les moyens à la disposition des membres de comités de retraite, soit les libérations pendant les heures de travail ${ }^{7}$, les capacités de formation continue et l'expertise interne, sont généralement insuffisants pour assurer le respect de la responsabilité fiduciaire. Le recours aux différentes formes de délégation et à l'expertise externe apparait également comme une solution face au manque de ressources.

Les questionnaires ont aussi fait ressortir plusieurs éléments intéressants au niveau du fonctionnement des comités. La grande majorité des régimes tiennent moins de cinq réunions par année (77\%). Ce sont les comités des régimes de plus grande taille qui tiennent le plus de réunions. La taille de la majorité des comités se situe entre six et neuf personnes. Ce sont uniquement les régimes de moins de 500 participants qui ont des comités de moins de 6 personnes.

La forte proportion de comités de retraite qui comporte plus de 10 membres s'explique par la conjonction de l'obligation légale de représentation des participants et de la représentation des différents groupes de participants. À partir des résultats du questionnaire, on a noté que les comités de grande taille sont associés à l'existence de plusieurs groupes professionnels à l'intérieur du même régime de retraite. On peut ainsi noter la forte proportion de répondants qui ont indiqué un régime unique dans leur organisation.

La $L o i R C R$ indique que tous les membres du comité de retraite sont solidairement responsables des décisions prises, à moins d'avoir exprimé clairement leur dissidence par une inscription au procès-verbal de la réunion. Dans l'enquête que nous avons menée, il est apparu que les décisions sont prises exceptionnellement par un vote. La règle est plutôt celle de l'unanimité. C'est même souvent une condition nécessaire pour prendre une décision. Dans toutes nos entrevues, un seul membre participant représentant les participants actifs a admis avoir utilisé une dissidence lors d'une réunion. 


\section{La gouvernance des régimes à cotisations déterminées}

La $L o i R C R$ a été rédigé à une époque où les régimes $C D$ étaient relativement peu présents au Québec ${ }^{8}$. Ces derniers étaient généralement des régimes de petite taille avec peu de responsabilités envers les participants actifs et inactifs. Au début des années 2000, le risque accru de conversion des régimes à prestations déterminées en régimes à cotisations déterminées a augmenté les sources de tensions entre la responsabilité fiduciaire des comités de retraite et la structure de gestion de ces régimes, qui comportent souvent des choix individualisés du niveau de cotisation et du profil des placements. De plus, la gestion des régimes $C D$ est généralement confiée à une institution financière qui choisit elle-même les placements et offre aux participants ses propres «formules» de rentes.

La première source de difficultés survient lorsque le régime CD est basé sur la participation volontaire des employés (que ce soit pour l'adhésion au régime ou pour le niveau de cotisations). Pour l'acteur syndical qui représente les employés, il faut alors investir beaucoup de ressources pour encourager la participation des employés. Cela passe par des outils d'information spécifiques, mais également par des assemblées syndicales et même par des « représentants » du régime dans les différents services de l'établissement ou de l'entreprise. Plusieurs entrevues ont fait ressortir l'importance d'un «leadership» individuel fort au sein du syndicat pour négocier et mettre en place le régime de retraite. La relève est difficile et il est même possible que certains régimes disparaissent lorsque le leader prendra sa retraite, par manque d'intérêts des plus jeunes travailleurs.

La seconde source de difficultés dans la gouvernance de ces régimes réside dans l'application de la responsabilité fiduciaire du comité de retraite. Le comité doit superviser la délégation de gestion des actifs, dans un contexte où le régime délègue ces activités à des institutions financières. La responsabilité fiduciaire est ainsi plus difficile à interpréter pour le comité de retraite. De plus, la possibilité qui existe fréquemment dans ce type de régime de gérer (souvent partiellement) individuellement ses placements rend plus difficile la participation aux assemblées annuelles et le recrutement de nouveaux membres pour le comité de retraite. Le comité de retraite a donc un pouvoir de supervision très limité, même s'il y a la même responsabilité fiduciaire que dans les régimes $P D$. Les régimes $C D$ demandent également plus de ressources pour informer et conseiller directement chacun des participants des enjeux de la planification financière pour la retraite. Certains participants aux entrevues se sont dit mal à l'aise avec cette responsabilité, alors que d'autres ne l'étaient pas, malgré le fait qu'ils n'avaient pas toujours de formation adéquate.

La troisième source de difficultés se situe au moment du départ à la retraite. Dans la plupart de ces régimes, les personnes à la retraite quittent le régime avec 
un capital individuel. Les parties (patronale ou syndicale) sont alors davantage sollicitées pour offrir des conseils aux membres, alors même que ce n'est pas leur rôle. Par ailleurs, ce type de régime rend la représentation des retraités au sein des comités de retraite problématique, dès lors qu'ils ne font plus partie du régime de retraite, mais que l'intérêt des retraités doit être représenté étant donné que les employés sont aussi de futurs retraités. La responsabilité de l'employeur, en tant que promoteur du régime, est également plus importante.

Face aux difficultés rencontrées dans la gouvernance des régimes à cotisations déterminées, les représentants des participants actifs bénéficient parfois des services de formation et de négociation offerts par les centrales syndicales au Québec. Cependant, il existe une tension entre la réponse aux besoins de services exprimés par les membres et l'orientation syndicale qui privilégie la promotion des régimes à prestations déterminées (FTQ, 2010; CSN, 2011). Les entrevues menées auprès de membres de comité de retraite de régimes $C D$ soulèvent des interrogations qui dépassent la recherche sur les conflits de rôle et qui concernent l'application même de la législation sur les régimes complémentaires de retraite. Pourtant, nous avons pu identifier et analyser les sources de tension spécifiques à la gouvernance des régimes à cotisations déterminées dans un contexte institutionnel forgé pour le cas-type des régimes à prestations déterminées.

\section{Discussion et conclusion}

Notre étude porte sur les conflits de rôle dans la gouvernance des régimes de retraite au Québec. Nous avons utilisé une méthodologie mixte, à la fois par questionnaire et par entrevue, afin de pouvoir contextualiser l'expérience des membres de comités de retraite et faire le lien entre les enjeux structurels des régimes de retraite et le fonctionnement habituel des comités. Face à une littérature périphérique sur le sujet et compte tenu des particularités québécoises, nous voulions mesurer les conflits de rôles potentiels de l'utilisation d'un comité de retraite comme outil de gouvernance des régimes de retraite.

Contrairement à nos anticipations, notre étude ne démontre pas de conflits systématiques causés par la coexistence du système de relations du travail et de la responsabilité fiduciaire des régimes complémentaires de retraite. Les tensions reliées aux relations du travail sont minimes. Nous avons bien identifié quelques enjeux, notamment au niveau du financement des régimes, de la viabilité des régimes qui relève de la confusion des rôles, toutefois, ces éléments ne sont pas perçus comme des sources de tensions, mais ils sont plutôt vus comme des enjeux à discuter. Le financement et la viabilité du régime constituent des enjeux pour les parties, et non pour les fiduciaires, mais quand ce sont les mêmes personnes qui discutent, il peut y avoir confusion de rôles. Toutefois, nos entrevues indiquent 
que les discussions au niveau de ces enjeux se font à l'extérieur des réunions de comités de retraite.

Des études précédentes sur le sujet mettaient l'emphase sur les conflits d'intérêts potentiels liés à la présence de fiduciaires membres de la direction (Cocco et Volpin, 2007). Le Québec est différent, car les fiduciaires proviennent aussi des salariés, retraités et/ou syndicats. Contrairement au résultat de Cocco et Volpin, le conflit d'intérêts créé par la présence de membres de la direction semble mitigé au Québec à cause de la provenance diversifiée des membres, ce qui pourrait expliquer les faibles tensions identifiées.

À certains égards, notre recherche corrobore les résultats d'études précédentes, notamment ceux de Kakabadse, Kakabadse et Kouzmin (2003), qui démontrent une cohérence dans la perception du rôle de fiduciaire, malgré la diversité démographique et l'expérience professionnelle. Notre étude n'indique pas de conflits systématiques et démontre que les décisions au niveau des comités de retraite du Québec sont prises à l'unanimité sans vote, malgré la diversité des membres du comité. Sayce (2009) indique que le facteur le plus important pour la participation à un comité de retraite est la volonté d'avoir une contribution utile. Bien que nous n'ayons pas posé spécifiquement cette question, le leadership exprimé par les participants à nos entrevues afin de mettre en place un régime de retraite s'inscrit dans les résultats de cette étude. Contrairement à d'autres études qui démontrent le processus politique de nomination des membres (Sayce et al., 2014), la particularité du Québec fait en sorte que les membres doivent provenir spécifiquement de certains groupes. Nos résultats indiquent, cependant, un problème de recrutement des membres actifs et inactifs, plutôt que des problèmes politiques de nomination.

Notre recherche représente une contribution à une meilleure connaissance du fonctionnement des régimes de retraite et de la mise en œuvre concrète de la Loi $R C R$ du point de vue des membres de comités de retraite. Elle a également montré que la gouvernance des régimes à cotisations déterminées, et dans une certaine mesure des régimes hybrides, représente un sujet de recherche particulier, autant du point de vue des relations du travail, que de la gouvernance des régimes de retraite.

Notre étude identifie certaines pistes pouvant améliorer la gouvernance des régimes de retraite au Québec. Nous sommes d'avis qu'il faudrait poursuivre les recherches sur l'efficacité de la $L o i R C R$ dans le but de permettre d'autres modalités de communication entre les membres du comité de retraite et les participants, ce qui favoriserait une plus grande participation des membres. La formation continue et l'acquisition d'opinions complémentaires est souvent une responsabilité assumée individuellement. L'offre, la proximité et la pertinence de ces formations semblent problématique pour les fiduciaires des régimes. De plus, 
le fort pourcentage (94 sur 204) des répondants qui désiraient «témoigner» de leur expérience peut indiquer un besoin de réflexivité et de communication des enjeux et des sources de tension au sein des comités de retraite. En effet, il n'existe pas de mécanisme systématique de consultation des membres de comités de retraite, en dehors d'évènements organisés dans le cadre de la formation syndicale ou par des associations professionnelles.

\section{Notes}

1 Actuellement, ce régime assure $25 \%$ du revenu gagné à tous les participants, jusqu'à un maximum du revenu admissible qui se situe à 54900 \$ en 2016. Une personne ayant travaillé toute sa carrière pourrait donc recevoir un maximum d'environ $13110 \$$ par an basé sur les paramètres de 2016.

2 Voir le texte de la Loi sur les régimes complémentaires de retraite, disponible à l'adresse suivante: http://www2.publicationsduquebec.gouv.qc.ca/dynamicSearch/telecharge.php?type=2 \&file=/R_15_1/R15_1.html

3 Le régime hybride comprend normalement les deux composantes, une partie à $C D$ et une partie à PD. Récemment, une nouvelle formule hybride est apparue, soit les régimes à prestations cibles. II s'agit d'un régime PD où les travailleurs se font promettre une rente de retraite (cible), mais cette dernière peut varier en fonction de la santé financière du régime.

4 II est impossible de prendre en compte la participation d'un même individu à plusieurs comités de retraite, même si cela semble assez courant.

5 Les régimes les plus importants, ceux des employés de la fonction publique (provinciale et fédérale) et du parapublique, des commissions scolaires, des policiers provinciaux, du secteur de la construction ne relèvent pas de la gestion de Retraite Québec et ne sont pas représentés dans la population de départ de l'enquête.

6 Les répondants assument, dans certains cas, plusieurs fonctions.

7 Le rapport du comité de travail présidé par Gilles Bernier recommandait d'inscrire, dans la Loi $R C R$, une obligation de libérations pour la formation continue des membres de comités de retraite (Bernier et al., 2006, p. 14).

8 Selon les statistiques de Retraite Québec, en 1990, au moment de l'adoption de la Loi RCR, il y avait 68336 personnes couvertes par des régimes à cotisations déterminées au Québec, contre 1322960 personnes couvertes par des régimes à prestations déterminées. En 2003, la première catégorie avait plus que doublé pour atteindre 141555 , alors que la seconde avait baissée à 1254978 .

\section{Bibliographie}

Bernier, Gilles, Louise Dagneaut, Jean Des Trois Maisons, Jean-Claude Dorval, Michel Lizée, Michel Méthot et Martin Rochette. (2006). Rôle des comités de retraite dans les régimes complémentaires de retraite et surveillance de la Régie des rentes du Québec. Tiré de http:// www.rrq.gouv.qc.ca/SiteCollectionDocuments/www.rrq.gouv.qc/Francais/publications/rcr/ rapport_comite_experts.pdf (consulté le 10 janvier 2017).

Biddle, Bruce J. (1979). Role Theory: Concepts and Research, New York: Krieger Pub Co.

Carmichael, Isla. (2005). Pension Power: Unions, Pension Funds, and Social Investment in Canada, Toronto: University of Toronto Press. 
Clark, Gordon L., Emiko Caerlewy-Smith et John C. Marshall. (2007). "The Consistency of UK Pension Fund Trustee Decision-Making». Journal of Pension Economics and Finance, 6 (1), p. 67-86.

Cocco, Joao F. et Paolo F. Volpin. (2007). "Corporate Governance of Pension Plans: The UK Evidence». Financial Analysts Journal, 63 (1), p. 70-83.

Confédération des syndicats nationaux. (2011). Propositions adoptées par le 63e Congrès. Tiré de https://www.csn.qc.ca/wp-content/uploads/2016/05/2011-11-23_PV_ann-A_63e_congres_ CSN.pdf (consulté le 10 janvier 2017).

Evans, John, Michael Orszag et John Piggott. (2008). Pension Fund Governance, Northampton: Edward Elgar Publishing.

Fédération des travailleurs du Québec. (2010). Pour un Québec qui nous ressemble et qui

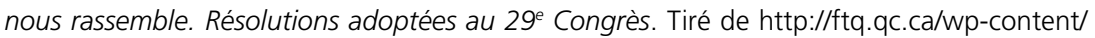
uploads/ftqimport/7229.pdf (consulté le 10 janvier 2017).

Fung, Archon, Tessa Hebb et Joel Rogers. (2001). Working Capital: The Power of Labor's Pensions, Ithaca, NY: Cornell University Press.

Gouvernement du Québec. (2013). Innover pour pérenniser le système de retraite: un contrat social pour renforcer la sécurité financière de tous les travailleurs québécois. Comité d'experts sur l'avenir du système de retraite québécois, 233 pages. Tiré de http://www.rrq.gouv.qc.ca/ SiteCollectionDocuments/www.rrq.gouv.qc/Francais/publications/rapport_comite/Rapport. pdf (consulté le 10 janvier 2017).

Hawley, James P., Andreas G. F. Hoepner, Keith L. Johnson, Joakim Sandberg et Edward J. Waitzer. (2014). Cambridge Handbook of Institutional Investment and Fiduciary Duty, Cambridge: Cambridge University Press.

Kakabadse, Nada, Andrew Kakabadse et Alex Kouzmin. (2003). "Pension Fund Trustees: Role and Contribution ». European Management Journal, 21 (3), p. 376-386.

Labrosse, Alexis. (2016). La présence syndicale au Québec en 2015. Tiré de https://www.travail. gouv.qc.ca/fileadmin/fichiers/Documents/presence_syndicale/2015.pdf (consulté le 10 janvier 2017).

Lizée, Michel. (2002). "Les enjeux des régimes de retraite en regard de l'action des syndicats québécois: couverture, sécurité du revenu et gestion des caisses de retraite ». Revue de l'IRES, 40 (3), p. 1-33. Tiré de http://www.ires-fr.org/publications-de-l-ires/item/2744-les-enjeuxdes-regimes-de-retraite-en-regard-de-l-action-des-syndicats-quebecois-couverture-securitedu-revenu-et-gestion-des-caisses-de-retraite (consulté le 10 janvier 2017).

McGill, Dan Mays. (2005). Fundamentals of Private Pensions, Oxford : Oxford University Press.

Pensions Expert Commission et Harry W. Arthurs. (2008). A Fine Balance: Safe PensionsAffordable Plans-Fair Rules, Toronto: Governement de l'Ontario.

Royal, Louise et André Brassard. (2010). "Comprendre les tensions de rôles afin de mieux les prévenir et de contribuer au bien-être des employés». Gestion, 35 (3), p. 27-33.

Sayce, Susan. (2009). Motivation and Pension Trusteeship Survey Report, Report prepared for the Centre for Diversity and Equality in Careers and Employment Research Report, Norwich: University of East Anglia.

Sayce, Susan, Johanna Weststar et Anil Verma. (2014). "The Recruitment and Selection of Pension Trustees: An Integrative Approach». Management Journal, 0143831X10387838. 


\section{RÉSUMÉ}

\section{Gouvernance des régimes complémentaires de retraite, relations du travail et conflits de rôle: une enquête québécoise}

Cette recherche vise à étudier la présence de conflits de rôle au sein des comités de retraite au Québec. Elle met en relation la gouvernance des régimes de retraite et la présence de conflits de rôle entre la représentation collective et la responsabilité fiduciaire du point de vue des membres de comités de retraite. Nous exploitons une particularité de la gestion québécoise, soit l'obligation de créer un comité de retraite sur lequel siègent des membres représentant l'employeur, les syndicats, les travailleurs, les retraités, ainsi que des membres indépendants. À l'aide d'une méthodologie mixte par questionnaire et par entrevue, il a été possible d'analyser les sources et la nature des conflits.

Contrairement à nos anticipations, notre étude ne démontre pas de conflits systématiques causés par la coexistence du système de relations du travail et de la responsabilité fiduciaire des régimes complémentaires de retraite. Les tensions reliées aux relations du travail sont minimes. Nous avons bien identifié quelques enjeux, notamment au niveau du financement des régimes, de la viabilité des régimes, qui relèvent de la confusion des rôles.

Toutefois ces éléments ne sont pas perçus comme des sources de tensions, mais plutôt comme des enjeux à discuter. La qualité de la gouvernance des régimes de retraite dépend des ressources investies par les acteurs des relations du travail dans l'accompagnement des membres des comités de retraite. La recherche sur les conflits de rôle dans la gouvernance des régimes de retraite au Québec montre que la gestion de ces conflits dépend, dans une large mesure, de la qualité de la législation sur l'administration des régimes complémentaires de retraite au Québec et, également, des ressources consacrées à la formation des membres des comités de retraite et des participants aux relations du travail.

MOTS-CLÉS: Québec, représentation collective, gouvernance, régime de retraite, responsabilité fiduciaire, conflit de rôle.

\section{SUMMARY}

\section{Governance of Supplementary Pension Plans, Labour Relations and Role Conflicts: A Quebec Study}

This research aims to study the presence of role conflict in pension committees in Quebec. It relates the governance of pension plans to the presence of role conflict between collective representation and fiduciary responsibility from the point of view of pension committee members. We exploit a particular feature of Quebec's pension funds legislation, namely the obligation to create a pension committee, which usually includes members representing the employer, trade unions, workers, retirees and independent members. Using a mixed methodology 
with a questionnaire and interviews, it was possible to analyse the sources and nature of these conflicts.

Contrary to our expectations, our study does not reveal systematic conflicts caused by the coexistence of a system of labour relations and the fiduciary responsibility of supplementary pension plans. Tensions related to labour relations are minimal. We have, however, identified a number of issues, notably relating to plan funding and plan sustainability, which leads to some role confusion.

That said, these aspects are not perceived as sources of tension, but are rather issues to be discussed. The quality of pension plans governance depends on the resources invested by labour relations collective actors in assisting members of pension committees. Our research on role conflict in the governance of pension plans in Quebec shows that the management of these conflicts depends to a large extent not only on the quality of the legislation governing the administration of supplemental pension plans, but also on the resources invested in training for retirement committee members.

KEYWORDS: Quebec, pension plans governance, labour relations, fiduciary responsibility, role conflict.

\section{RESUMEN}

\section{Gobernanza de regímenes complementarios de jubilación, relaciones laborales y conflictos de rol: un estudio quebequense}

Esta investigación se propone estudiar la presencia de conflictos de rol en el seno de los comités de jubilación en la provincia de Quebec. Se pone en relación la gobernanza de los regímenes de jubilación y la presencia de conflictos de rol entre la representación colectiva y la responsabilidad fiduciaria desde el punto de vista de los miembros de los comités de jubilación. Explotamos una particularidad de la gestión quebequense, es decir la obligación de crear un comité de jubilación en el cual deben participar los miembros representantes del empleador, de los sindicatos, de los trabajadores, de los jubilados y de los miembros independientes. Apoyándonos en una metodología mixta, con cuestionarios y entrevistas, se pudo analizar las fuentes y la naturaleza de los conflictos.

Al contrario de nuestras anticipaciones, nuestro estudio no mostró la presencia de conflictos sistemáticos causados por la coexistencia del sistema de relaciones laborales y de la responsabilidad fiduciaria de los regímenes complementarios de jubilación. Las tensiones vinculadas a las relaciones laborales son mínimas. Se identificaron algunas cuestiones, en particular relacionadas al financiamiento de los regímenes y de la viabilidad de dichos regímenes, vinculados a la confusión de roles.

Sin embargo, estos elementos no son percibidos como fuentes de tensión sino más bien como cuestiones a discutir. La calidad de la gobernanza de los regímenes 
de jubilación depende de los recursos invertidos por los actores de las relaciones laborales en el acompañamiento de los miembros de los comités de jubilación. La investigación sobre los conflictos de rol en la gobernanza de los regímenes de jubilación en Quebec muestra que la gestión de dichos conflictos depende, en gran parte, de la calidad de la legislación en materia de administración de regímenes complementarios de jubilación en Quebec y, igualmente, de los recursos consagrados a la formación de los miembros de los comités de jubilación y de los participantes a las relaciones laborales.

PALABRAS CLAVES: Quebec, representación colectiva, gobernanza, régimen de jubilación, responsabilidad fiduciaria, conflicto de rol. 